\title{
Dynamic sensitivity of photon-dressed atomic ensemble with quantum criticality
}

\author{
Jin-Feng Huang (黄金风), ${ }^{1}$ Yong Li (李勇), ${ }^{2}$ Jie-Qiao Liao (廖洁桥), ${ }^{3}$ Le-Man Kuang (匡乐满), ${ }^{1, *}$ and C. P. Sun (孙昌璞) ${ }^{3, \dagger}$ \\ ${ }^{1}$ Key Laboratory of Low-Dimensional Quantum Structures and Quantum Control of Ministry of Education \\ and Department of Physics, Hunan Normal University, Changsha 410081, China \\ ${ }^{2}$ Department of Physics, The University of Hong Kong, Pokfulam Road, Hong Kong, China \\ ${ }^{3}$ Institute of Theoretical Physics, Chinese Academy of Sciences, Beijing 100190, China
}

(Received 15 February 2009; published 10 December 2009)

\begin{abstract}
We study the dynamic sensitivity of an atomic ensemble dressed by a single-mode cavity field (called a photon-dressed atomic ensemble), which is described by the Dicke model near the quantum critical point. It is shown that when an extra atom in a pure initial state passes through the cavity, the photon-dressed atomic ensemble will experience a quantum phase transition showing an explicit sudden change in its dynamics characterized by the Loschmidt echo of this quantum critical system. With such dynamic sensitivity, the Dicke model can resemble the cloud chamber for detecting a flying particle by the enhanced trajectory due to the classical phase transition.
\end{abstract}

DOI: 10.1103/PhysRevA.80.063829

PACS number(s): 42.50.Nn, 73.43.Nq, 03.65.Yz

\section{INTRODUCTION}

The quantum phase transition $(\mathrm{QPT})[1]$ occurs at zero temperature when the external parameters of some interacting many-body systems change to reach the critical values. Generally, it is associated with the ground state with energy level crossing and symmetry breaking at the critical points. Recently, it was discovered that near the quantum critical point the QPT system possesses the ultrasensitivity in its dynamical evolution [2]. This theoretical prediction has been demonstrated by an NMR experiment [3]. Similar sensitivity exists in some quantum systems [4-9] possessing QPT.

In this paper, we study the dynamic sensitivity of an atomic ensemble in a cavity with a single-mode electromagnetic field (called a photon-dressed atomic ensemble), which is described by the Dicke model [10]. We assume the atoms in the Dicke model are resonant with the cavity field. When an extra two-level atom in large detuning goes through the cavity field, the frequency of cavity field will be shifted effectively according to the Stark effect so that the photondressed atomic ensemble near the QPT will be forced into its critical point. In this situation the dynamic evolution of the Dicke model becomes too sensitive in response to the passage of the extra atom.

Here, this dynamic sensitivity is characterized by the Loschmidt echo (LE) [11], which is intrinsically defined by the structure of the photon-dressed atomic ensemble. For a short-time approximation, we prove that the LE is just an exponential function of the photon number variance in the photon-dressed atomic ensemble. This finding means that the LE can be experimentally measured by detecting the photon correlation. Its sudden change may imply the passage of an extra atom through the cavity. With this reorganization we will demonstrate that such quantum sensitivity in the Dicke model is very similar to the classical sensitivity of the cloud chamber for detecting a flying particle, which is character-

\footnotetext{
*1mkuang@hunnu.edu.cn

†suncp@itp.ac.cn; http://www.itp.ac.cn/ suncp
}

ized by the macroscopically observable trajectories enhanced by the classical phase transitions.

This paper is organized as follows. In Sec. II, we describe the setup of the quantum critical model based on the Dicke model. The effective Hamiltonian is given in terms of the collective excitation of the atomic ensemble. Then the analytic calculation of LE (or the decoherence of the extra atom) is carried out in Sec. III for the normal and super-radiant phases, respectively, by short-time approximation. In the following Sec. IV we plot some figures to explicitly show the sensitive properties of the LE. In Sec. V, we address the similarity between the dynamic sensitivity of the photondressed atomic ensemble induced by an extra atom and the classical cloud chamber. Finally, we draw our conclusion in Sec. VI. The detailed coefficients for Bogoliubov transformation in Sec. IV are given in the Appendix.

\section{MODEL AND HAMILTONIAN}

As showed in Fig. 1, we consider an atomic ensemble confined in a gas cell coupled with a single-mode cavity field of frequency $\omega$, which is described by the annihilation (creation) operator $a\left(a^{\dagger}\right)$. We use the Pauli matrices

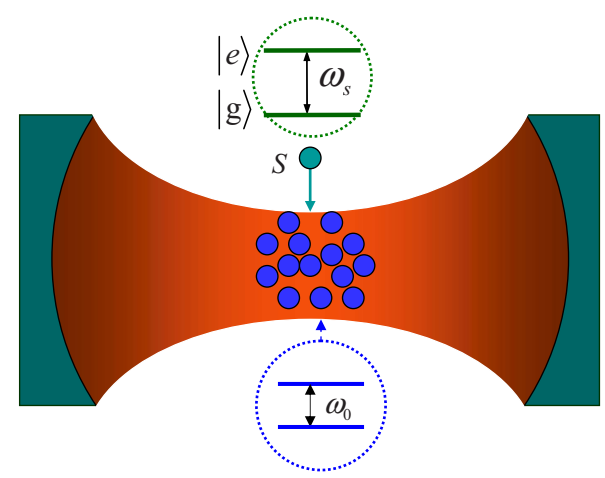

FIG. 1. (Color online) Schematic of a cavity field coupled with an atomic gas consisting of $N$ two-level atoms. An extra detected two-level atom $S$ is injected into cavity field. 
$\sigma_{z}^{(j)}=|e\rangle_{j j}\langle e|-| g\rangle_{j j}\left\langle g\left|, \quad \sigma_{+}^{(j)}=\right| e\right\rangle_{j j}\langle g|$, and $\sigma_{-}^{(j)}=|g\rangle_{j j}\langle e|$ to describe the atomic transition of the $j$ th atom with energy level spacing $\omega_{0}$, where $|e\rangle_{j}$ and $|g\rangle_{j}$ are the excited and ground states of the $j$ th atom, respectively. The system of the atomic ensemble coupled with the single-mode cavity field is described by the Dicke model (hereafter, we take $\hbar=1$ ),

$$
H_{0}=\omega a^{\dagger} a+\sum_{j=1}^{N}\left[\frac{1}{2} \omega_{0} \sigma_{z}^{(j)}+g_{0}\left(a^{\dagger}+a\right)\left(\sigma_{-}^{(j)}+\sigma_{+}^{(j)}\right)\right] .
$$

Here, for small-dimension atomic gas [5], we have assumed that all the atoms locate near the origin point and interact with the cavity field with the identical coupling strength $g_{0}$.

An extra two-level atom $S$ with transition operators $\sigma_{z}$, $\sigma_{+}$, and $\sigma_{-}$couples to the original single-mode cavity field with Hamiltonian

$$
H_{I}=\frac{1}{2} \omega_{s} \sigma_{z}+g_{s}\left(a^{\dagger} \sigma_{-}+a \sigma_{+}\right)
$$

where we have made a rotating wave approximation. Similarly, $\omega_{s}$ is the transition frequency between the ground state $|g\rangle$ and excited state $|e\rangle$ of the atom $S ; g_{s}$ is the corresponding coupling strength.

It has been shown that the QPT will occur in the system described by Dicke Hamiltonian (1) [5], since it keeps Hermitian only for a small coupling strength $g_{0}$. But it is only a model to display QPT in quantum optical system. Actually it could not happen for the realistic atomic, molecular, and optical (AMO) system if the unreasonably ignored two-photon term $A^{2}$ is included [12]. To focus on our main idea in the work, we only regard the Dicke system as a simplified model. We would like to point out that many authors have recognized this problem, but there still exist many explorations by using this simplified model [13].

If the atom $S$ is far-off-resonant with the cavity field, that is, the detuning $\Delta_{s}\left(\equiv \omega_{s}-\omega\right)$ is much larger than the corresponding coupling strength $g_{s}$, i.e., $\left|\Delta_{s}\right| \gg g_{s}$, then one can use the so-called Fröhlich-Nakajima transformation $[14,15]$ (or other elimination methods) to obtain the effective total Hamiltonian

$$
\begin{aligned}
H_{\mathrm{eff}}= & \left(\omega+\widetilde{\delta} \sigma_{z}\right) a^{\dagger} a+\frac{1}{2}\left(\omega_{s}+\widetilde{\delta}\right) \sigma_{z}+\frac{\omega_{0}}{2} \sum_{j=1}^{N} \sigma_{z}^{(j)} \\
& +\frac{g}{\sqrt{N}} \sum_{j=1}^{N}\left(a^{\dagger}+a\right)\left(\sigma_{-}^{(j)}+\sigma_{+}^{(j)}\right),
\end{aligned}
$$

where $\tilde{\delta} \equiv g_{s}^{2} / \Delta_{s}$ and $g \equiv g_{0} \sqrt{N}$. We note that the FröhlichNakajima transformation is equivalent to the approach based on the adiabatical elimination.

The Hilbert space of $N$ two-level atoms is spanned by $2^{N}$ basis states. In the current case all the atoms have the same free frequencies and coupling constants with the cavity field, we can consider these atoms being identical. Then the Hilbert space is reduced into a subspace of $(2 N+1)$ dimension. In this subspace, Hamiltonian (3) is simplified by introducing the collective atomic operators

$$
J_{ \pm}=\sum_{j=1}^{N} \sigma_{ \pm}^{(j)}, \quad J_{z}=\frac{1}{2} \sum_{j=1}^{N} \sigma_{z}^{(j)},
$$

which obey the following angular momentum commutation relations:

$$
\left[J_{z}, J_{ \pm}\right]= \pm J_{ \pm}, \quad\left[J_{+}, J_{-}\right]=2 J_{z} .
$$

The collective atomic operator $J_{z}$ denotes the collective population of the atomic gas and $J_{ \pm}$represents the collective transitions.

In terms of the above angular momentum operators, Hamiltonian (3) is written as

$$
\begin{aligned}
H_{\mathrm{eff}}= & \left(\omega+\widetilde{\delta} \sigma_{z}\right) a^{\dagger} a+\frac{1}{2}\left(\omega_{s}+\widetilde{\delta}\right) \sigma_{z}+\omega_{0} J_{z} \\
& +\frac{g}{\sqrt{N}}\left(a^{\dagger}+a\right)\left(J_{+}+J_{-}\right),
\end{aligned}
$$

which is further reduced to

$$
\begin{aligned}
H_{\mathrm{eff}}= & \left(\omega+\tilde{\delta} \sigma_{z}\right) a^{\dagger} a+\omega_{0} b^{\dagger} b+\frac{1}{2}\left(\omega_{s}+\tilde{\delta}\right) \sigma_{z}+g\left(a^{\dagger}+a\right) \\
& \times\left(b^{\dagger} \sqrt{1-b^{\dagger} b / N}+\text { H.c. }\right)
\end{aligned}
$$

(up to constant terms) through making use of the HolsteinPrimakoff [16] transformation, which represents the angular momentum operators in terms of a single bosonic mode as follows:

$$
\begin{gathered}
J_{+}=b^{\dagger} \sqrt{N-b^{\dagger} b}, \\
J_{-}=\sqrt{N-b^{\dagger} b} b, \\
J_{z}=b^{\dagger} b-\frac{1}{2} N .
\end{gathered}
$$

To see more explicitly the dynamic sensitivity of the photon-dressed atomic ensemble in response to the extra atom, corresponding to different state of the extra atom, the effective Hamiltonian in Eq. (7) reads

$$
H_{\text {eff }}=|g\rangle\left\langle g\left|\otimes H_{g}+\right| e\right\rangle\langle e| \otimes H_{e},
$$

with

$$
H_{g}=\omega_{g} a^{\dagger} a+\omega_{0} b^{\dagger} b+g\left(a^{\dagger}+a\right)\left(b^{\dagger} \sqrt{1-b^{\dagger} b / N}+\text { H.c. }\right),
$$

$$
H_{e}=\omega_{e} a^{\dagger} a+\omega_{0} b^{\dagger} b+g\left(a^{\dagger}+a\right)\left(b^{\dagger} \sqrt{1-b^{\dagger} b / N}+\text { H.c. }\right),
$$

where $\omega_{e}=\omega+\widetilde{\delta}$ and $\omega_{g}=\omega-\widetilde{\delta}$. Note that in the derivation of the above Hamiltonians (10) and (11), we have discarded some constant terms.

\section{QUANTUM CRITICAL EFFECT}

Before the extra atom $S$ is sent into the cavity, the photondressed atomic ensemble (including the cavity field and the 
atomic gas) is described by the Dicke Hamiltonian

$$
H_{G}=\omega a^{\dagger} a+\omega_{0} b^{\dagger} b+g\left(a^{\dagger}+a\right)\left(b^{\dagger} \sqrt{1-b^{\dagger} b / N}+\text { H.c. }\right) \text {. }
$$

Comparing Eqs. (10) and (11) with Eq. (12), we find, as a result of the injection of the atom $S$, only the frequency of the optical field changes by a small shift $\tilde{\delta}$ in the dynamic evolution of the photon-dressed atomic ensemble.

The photon-dressed atomic ensemble is initially prepared in the ground state $|G\rangle$ of Hamiltonian (12) and the extra atom $S$ in a superposed state $\alpha|g\rangle+\beta|e\rangle$, where the normalization condition requires $|\alpha|^{2}+|\beta|^{2}=1$. When the extra atom $S$ interacts dispersively with the cavity field, the total system is governed by Hamiltonians (10) and (11) corresponding to the extra atom $S$ in states $|g\rangle$ and $|e\rangle$, respectively. Then at time $t$ the state of the total system becomes an entanglement one,

$$
\begin{aligned}
|\Psi(t)\rangle & =e^{-i H_{\text {eff }} t}(\alpha|g\rangle+\beta|e\rangle) \otimes|G\rangle \\
& =\alpha|g\rangle \otimes e^{-i H_{g} t}|G\rangle+\beta|e\rangle \otimes e^{-i H_{e} t}|G\rangle \\
& \equiv \alpha|g\rangle \otimes\left|G_{g}(t)\right\rangle+\beta|e\rangle \otimes\left|G_{e}(t)\right\rangle,
\end{aligned}
$$

where we have defined

$$
\left|G_{g}(t)\right\rangle \equiv e^{-i H_{g} t}|G\rangle, \quad\left|G_{e}(t)\right\rangle \equiv e^{-i H_{e} t}|G\rangle .
$$

The generation of the above entanglement is due to the conditional dynamics of the total system. This is to say, corresponding to the detected atom prepared in states $|g\rangle$ and $|e\rangle$, the evolution of the photon-dressed atomic ensemble will be governed by the Hamiltonians $H_{g}$ and $H_{e}$, respectively. The central task of this paper is to show that the dynamic of the photon-dressed atomic ensemble is sensitive to the state of the extra atom. When the photon-dressed atomic ensemble stays in the vicinity of the QPT, the effect of QPT must impose on the state of the extra atom with some enhancement fashion, like the results in Ref. [2]. This motivates us to study the quantum decoherence of the extra atom near the critical point of the photon-dressed atomic ensemble, which can also reflect the dynamic sensitivity of the photon-dressed atomic ensemble.

By tracing over the degree of freedom of the photondressed atomic ensemble in evolution state (13), the reduced density matrix $\rho_{s}(t)=\operatorname{Tr}_{a, b}\{|\Psi(t)\rangle\langle\Psi(t)|\}$ of the detected atom is obtained as

$$
\rho_{s}(t)=|\alpha|^{2}|g\rangle\left\langle\left. g|+| \beta\right|^{2} \mid e\right\rangle\langle e|+\left(D \alpha^{*} \beta|e\rangle\langle g|+\text { H.c. }\right),
$$

where we have introduced the decoherence factor

$$
D(t)=\left\langle G\left|\exp \left(i H_{g} t\right) \exp \left(-i H_{e} t\right)\right| G\right\rangle .
$$

Alternatively, we can investigate the decoherence of the extra atom by examining the so-called LE

$$
L(t) \equiv|D(t)|^{2}
$$

defined for the dynamic sensitivity of the photon-dressed atomic ensemble. For a short time $t$, the LE can be approximated as

$$
L(t) \approx\left|\left\langle G\left|e^{-2 i \tilde{\delta} t a^{\dagger} a}\right| G\right\rangle\right|^{2} .
$$

The straightforward calculation can give

$$
L(t) \approx \exp \left(-4 \gamma \widetilde{\delta}^{2} t^{2}\right) .
$$

Here, we have introduced the photon number variance

$$
\gamma \equiv\left\langle\left(a^{\dagger} a\right)^{2}\right\rangle-\left\langle a^{\dagger} a\right\rangle^{2},
$$

and the average $\langle\cdot\rangle$ is taken for the ground state $|G\rangle$.

We point out that, up to the second order of time $t$, the decay rate of the LE depends not only on $t^{2}$, but also on the photon number variance $\gamma$. It is well known that the photondressed atomic ensemble described by Dicke Hamiltonian (12) transits from the normal phase to the super-radiant one with the increase in the parameter $g$ from that less than the critical value $g_{c}=\sqrt{\omega \omega_{0}} / 2$ to that larger than $g_{c}$. Going across the phase transition point, the ground state of the photondressed atomic ensemble experiences a complex change. We can predict that the photon number variance $\gamma$ of the ground state will exhibit some special features at the critical point.

According to Eq. (13), we can imagine that the quantum criticality of the photon-dressed atomic ensemble can display which single state $|g\rangle$ or $|e\rangle$ that the extra atom stays. When $L(t)$ approaches zero, the photon-dressed atomic ensemble is forced into two orthogonal states $\left|G_{g}(t)\right\rangle$ and $\left|G_{e}(t)\right\rangle$, and thus it behaves as a measurement apparatus to detect the state of the extra atom. In this case, its measurement on the atom will induce the decoherence of the extra atom. In what follows, we will calculate the photon number variance $\gamma$ of the photon-dressed atomic ensemble in two different phases, that is, the normal phase and the super-radiant phase.

\section{A. Dynamic sensitivity in normal phase}

In this subsection, we explicitly calculate $\gamma$ to investigate the properties of the LE when the photon-dressed atomic ensemble is within the normal phase. In the case of low excitations at thermodynamic limit $N \rightarrow \infty$, Hamiltonian (12) becomes

$$
H_{G}=\omega a^{\dagger} a+\omega_{0} b^{\dagger} b+g\left(a^{\dagger}+a\right)\left(b^{\dagger}+b\right)
$$

for $\sqrt{1-b^{\dagger} b / N} \approx 1$, which is typical to describe two-coupled harmonic oscillators. It is well known that Hamiltonian (21) becomes non-Hermitian in the overstrong coupling region $g>g_{c}$, namely, the Hamiltonian possesses imaginary eigenvalues [17]. This means effective Hamiltonian (21) is illdefined for $g>g_{c}$. Therefore, we now restrict the Hamiltonian within the so-called normal phase region $g<g_{c}$. Correspondingly, this limited Hamiltonian (21) describes the normal phase of the Dicke model.

In the normal phase, Hamiltonian (21) can be diagonalized as

$$
H_{G}=\omega_{A} A^{\dagger} A+\omega_{B} B^{\dagger} B
$$

by introducing the polariton operators $A\left(A^{\dagger}\right)$ and $B\left(B^{\dagger}\right)$, which depict the mixed bosonic fields of photons and collective atomic excitations. The eigenfrequencies of the polaritons $A$ and $B$ are 


$$
\begin{aligned}
& \omega_{A}^{2}=\frac{1}{2}\left(\omega_{0}^{2}+\omega^{2}\right)-\frac{1}{2} \sqrt{\left(\omega_{0}^{2}-\omega^{2}\right)^{2}+16 g^{2} \omega_{0} \omega}, \\
& \omega_{B}^{2}=\frac{1}{2}\left(\omega_{0}^{2}+\omega^{2}\right)+\frac{1}{2} \sqrt{\left(\omega_{0}^{2}-\omega^{2}\right)^{2}+16 g^{2} \omega_{0} \omega} .
\end{aligned}
$$

It is straightforward to see that $\omega_{A}^{2}<0$ when $g>g_{c} \equiv \sqrt{\omega \omega_{0}} / 2$. That is, the eigenfrequency $\omega_{A}$ of mode $A$ becomes a complex number, which means Hamiltonian (22) will be non-Hermitian in the coupling region of $g>g_{c}$.

The relations between the operators $\left\{a, b, a^{\dagger}, b^{\dagger}\right\}$ and $\left\{A, B, A^{\dagger}, B^{\dagger}\right\}$ are given by

$$
\begin{aligned}
& a^{\dagger}=f_{1} A^{\dagger}+f_{2} A+f_{3} B^{\dagger}+f_{4} B, \\
& b^{\dagger}=h_{1} A^{\dagger}+h_{2} A+h_{3} B^{\dagger}+h_{4} B,
\end{aligned}
$$

where the concrete forms of coefficients $f_{i}$ and $h_{i}(i=1,2,3,4)$ have been given by Ref. [5]. Here we only give the detailed forms of $f_{i}$ in the Appendix.

From Eq. (22), we can see that the ground state of the photon-dressed atomic ensemble in the polariton representation is $|G\rangle=|0\rangle_{A} \otimes|0\rangle_{B} \equiv|00\rangle_{A B}$. Making use of Eqs. (20) and (25), we can obtain the photon number variance

$$
\gamma=2 f_{1}^{2} f_{2}^{2}+2 f_{3}^{2} f_{4}^{2}+\left(f_{1} f_{4}+f_{2} f_{3}\right)^{2} .
$$

In the normal phase, all the coefficients $f_{i}(i=1,2,3,4)$ are real, then the photon variance is a positive number, which implies the coherence of the extra atom will vanish with time.

We have mentioned that Hamiltonian (21) of two-coupled harmonic oscillators cannot work well in the overstrong coupling region $\left(g>g_{c}\right)$. This is because the approximation $\sqrt{1-b^{\dagger} b / N} \approx 1$ for the original one [Eq. (12)] cannot make sense in this region. Thus, we need to consider a different approximation for Eq. (12) when $g>g_{c}$.

\section{B. Dynamic sensitivity in super-radiant phase}

Physically, when the atom-light coupling becomes stronger and stronger, the coupled system will acquire a macroscopic excitations of atomic ensemble. And then the system enters into a super-radiant phase when $g>g_{c}$. In this situation, the low-excitation approximation is no longer valid. We can use the coherent state $|\beta\rangle$ of the collective atomic operator $b$ to depict these kinds of macroscopic excitations [4]. To achieve the effective Hamiltonian over such background of macroscopic excitations, we need to do the displacement $[4,5]$

$$
b^{\dagger} \rightarrow b^{\prime \dagger}-\sqrt{\beta}
$$

(or alternatively, $b^{\dagger} \rightarrow b^{\prime \dagger}+\sqrt{\beta}$ ). Correspondingly, we also displace the optical field by

$$
a^{\dagger} \rightarrow a^{\prime \dagger}+\sqrt{\alpha}
$$

(or alternatively, $a^{\dagger} \rightarrow a^{\prime \dagger}-\sqrt{\alpha}$ ). Here $a^{\prime \dagger}$ and $b^{\prime \dagger}$ describe quantum fluctuations about the semiclassical steady state [18]; elsewhere, $\sqrt{\alpha}$ and $\sqrt{\beta}$ describe the macroscopic mean fields above $g_{c}$ in the order of $O(\sqrt{N})$ [5]. Then Hamiltonian (12) becomes

$$
\begin{aligned}
H_{G}= & \omega_{0}\left[b^{\prime \dagger} b^{\prime}-\sqrt{\beta}\left(b^{\prime \dagger}+b^{\prime}\right)+\beta\right]+\omega\left[a^{\prime \dagger} a^{\prime}+\sqrt{\alpha}\left(a^{\prime \dagger}+a\right)\right. \\
& +\alpha]+g \sqrt{\frac{k}{N}}\left(a^{\prime \dagger}+a^{\prime}+2 \sqrt{\alpha}\right)\left(b^{\prime \dagger} \sqrt{\xi}+\sqrt{\xi} b^{\prime}-2 \sqrt{\beta} \sqrt{\xi}\right),
\end{aligned}
$$

where

$$
\sqrt{\xi}=\sqrt{1-\left[d^{\dagger} d-\sqrt{\beta}\left(d^{\dagger}+d\right)\right] /(N-\beta)}
$$

is introduced. In the thermodynamic limit $N \rightarrow \infty$, for Eq. (29), we follow Emary and Brandes [5]: expand the square root $\sqrt{\xi}$ and keep terms up to the order of $N^{0}$ in the Hamiltonian. Then through choosing the appropriate displacements

$$
\sqrt{\alpha}=\frac{g}{\omega} \sqrt{N\left(1-\mu^{2}\right)}, \quad \sqrt{\beta}=\sqrt{\frac{N}{2}(1-\mu)}
$$

with $\mu=\omega \omega_{0} / 4 g^{2}$, we can diagonalize Hamiltonian (29) as

$$
H_{G}=\omega_{A}^{\prime} A^{\prime \dagger} A^{\prime}+\omega_{B}^{\prime} B^{\prime \dagger} B^{\prime}
$$

by the Bogoliubov transformation

$$
\begin{aligned}
& a^{\prime \dagger}=f_{1}^{\prime} A^{\prime \dagger}+f_{2}^{\prime} A^{\prime}+f_{3}^{\prime} B^{\prime \dagger}+f_{4}^{\prime} B^{\prime}, \\
& b^{\prime \dagger}=h_{1}^{\prime} A^{\prime \dagger}+h_{2}^{\prime} A^{\prime}+h_{3}^{\prime} B^{\prime \dagger}+h_{4}^{\prime} B^{\prime},
\end{aligned}
$$

where the coefficients $f_{i}^{\prime}$ and $h_{i}^{\prime}(i=1,2,3,4)$ have been given in Ref. [5]. Here we only give the detailed forms of $f_{i}^{\prime}$ in the Appendix.

The eigenfrequencies $\omega_{A}^{\prime}$ and $\omega_{B}^{\prime}$ of the polaritons described by the operators $A^{\prime}$ and $B^{\prime}$ are given by

$$
\begin{aligned}
& \omega_{A}^{\prime 2}=\frac{1}{2}\left[\frac{\omega_{0}^{2}}{\mu^{2}}+\omega^{2}-\sqrt{\left(\frac{\omega_{0}^{2}}{\mu^{2}}-\omega^{2}\right)^{2}+4 \omega^{2} \omega_{0}^{2}}\right], \\
& \omega_{B}^{\prime 2}=\frac{1}{2}\left[\frac{\omega_{0}^{2}}{\mu^{2}}+\omega^{2}+\sqrt{\left(\frac{\omega_{0}^{2}}{\mu^{2}}-\omega^{2}\right)^{2}+4 \omega^{2} \omega_{0}^{2}}\right] .
\end{aligned}
$$

It is known that if the coupling strength $g$ exceeds the critical value $g_{c}$, both the above eigenfrequencies are real, but not in the region of $g<g_{c}$. Namely, when $g>g_{c}$, Hamiltonian (30) is Hermitian.

In the super-radiant phase, the ground state $|G\rangle=|00\rangle_{A^{\prime} B^{\prime}}$ satisfies $A^{\prime}|G\rangle=B^{\prime}|G\rangle=0$. Similar to the normal phase, we can calculate the photon number variance in the super-radiate phase as

$$
\begin{aligned}
\gamma= & 2 f_{1}^{\prime 2} f_{2}^{\prime 2}+2 f_{3}^{\prime 2} f_{4}^{\prime 2}+\left(f_{1}^{\prime} f_{4}^{\prime}+f_{2}^{\prime} f_{3}^{\prime}\right)^{2} \\
& +\alpha\left[\left(f_{1}^{\prime}+f_{2}^{\prime}\right)^{2}+\left(f_{3}^{\prime}+f_{4}^{\prime}\right)^{2}\right] .
\end{aligned}
$$

Compared with the case of normal phase, the displacement $\alpha$ of the photon operator appears in the photon number variance.

\section{PHOTON NUMBER VARIANCE FOR LOSCHMIDT ECHO}

We have separately calculated the LE of the photondressed atom ensemble perturbed by an extra atom in two 


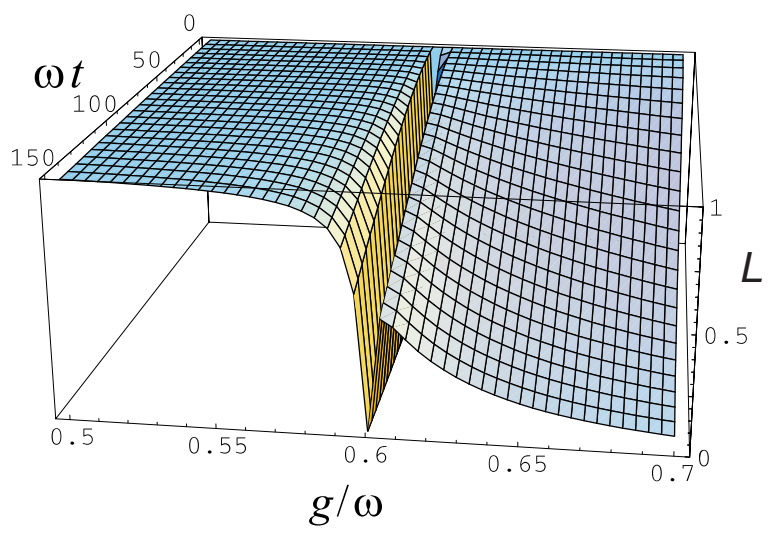

FIG. 2. (Color online) 3D diagram of the LE plotted as a function of the time $t$ and the coupling strength $g$ both in the normal phase (the left panel) and in the super-radiant phase (the right panel). Here, in unit of $\omega, \omega_{0}=1.44 \omega, \widetilde{\delta}=g_{s}^{2} / \Delta_{s}=0.001 \omega\left(\Delta_{s}\right.$ $\left.=0.1 \omega, g_{s}=0.01 \omega\right)$, the critical point $g_{c}=\sqrt{\omega \omega_{0}} / 2=0.6 \omega$, the number of atoms $N=100$.

quantum phases: normal phase and super-radiant phase. Our calculations are based on the short-time approximation, but it can cover the main character of the QPT of the photondressed atomic ensemble induced by the extra atom. As follows, we illustrate the LE versus the coupling strength $g$ and time $t$ by plotting its three-dimensional (3D) contour.

Figure 2 shows the LE as a function of the time $t$ and the coupling strength $g$ in the normal and super-radiant phases. It is obvious that the LE, which is calculated from Eqs. (19), (26), and (34), will have a sudden change near the critical point. Its decay is highly enhanced at the critical value $g_{c}$. In the normal phase, the LE decays rapidly to zero as the enlarged coupling strength $g$ of the photon-dressed atomic ensemble approaches the critical point $g_{c}$. In the super-radiant phase, similarly, the LE decays faster as the parameter $g$ decreases to the critical point $g_{c}$. Then the coherence of the extra atom is very sensitive to the dynamical perturbation of the photon-dressed atomic ensemble near the critical point.

Meanwhile, in the vicinity of the critical point, the coherence of the extra atom decreases to zero sharply with time at fixed point of $g$. The more nearly the work point $g$ approaches the critical point $g_{c}$, the sharper the decay of the decoherence of the extra atom is. During this process, the detected atom evolves from a pure state to a mixed one. Therefore, we can measure the QPT of the photon-dressed atomic ensemble by exploring the coherence of the detected atom in the photon-dressed atomic ensemble.

Figure 3 shows the LE at a fixed time $(\omega t=100)$ for the photon-dressed atomic ensemble in both the normal and super-radiant phases. Contrary to the case of the transverse field Ising model, the LE in the present system will not approach 1 when the coupling strength is much more than the critical point (seen from Fig. 3). The reason is that a large displacement $\sqrt{\alpha} \propto g \sqrt{N}$ appears in the super-radiant phase and will increase as the coupling strength increases. That means a small disparity $\left(\widetilde{\delta} a^{\dagger} a\right)$ in the initial Hamiltonian in the super-radiant phase may lead to a large difference (e.g., the decoherence factor will decay faster) after period of long-

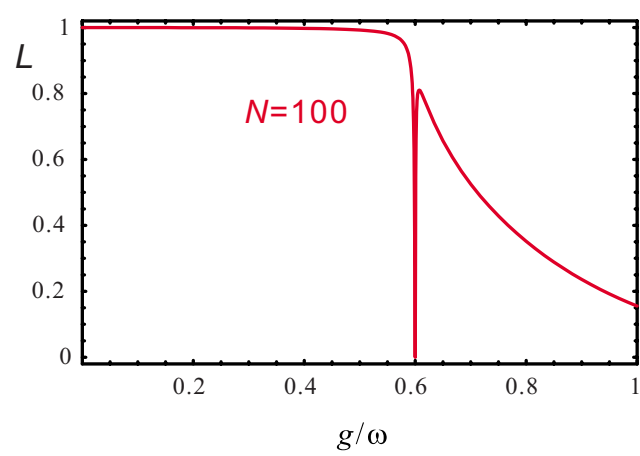

FIG. 3. (Color online) The cross section of the 3D surface of the LE in Fig. 2 at $\omega t=100$. For other parameters see Fig. 2.

enough time. As pointed out in Ref. [5], the so-called quantum chaos always appears in the super-radiant phase.

It follows from Eqs. (19), (26), and (A1) that the LE is independent of $N$ in the normal phase. However, the LE depends on the number of the atoms $N$ in the super-radiant phase via $\sqrt{\alpha} \propto \sqrt{N}$. In Fig. 4, the LE is plotted as a function of the coupling strength $g$ with $N=100,1000$, and 10000 respectively. It can be observed from Fig. 4 that the LE line decays faster and faster in the super-radiant phase as the atom number $N$ increases. The reason is the same as that mentioned above. The photon number variance $\gamma$ proportional to the decay rate for the decoherence of the extra atom increases as $N$ increases via approximately

$$
\gamma \propto \alpha \propto g^{2} N .
$$

Accordingly, the LE decreases with the form

$$
\ln L \propto-g^{2} N
$$

in the super-radiant phase. Thus, as $N \rightarrow \infty$, the decay of the LE will be strongly enhanced at the critical point.

\section{ANALOG TO CLOUD CHAMBER}

Now we can address the similarity of sensitive dynamics between the present system and the classical cloud chamber. In classical cloud chamber, when a charged particle (or a

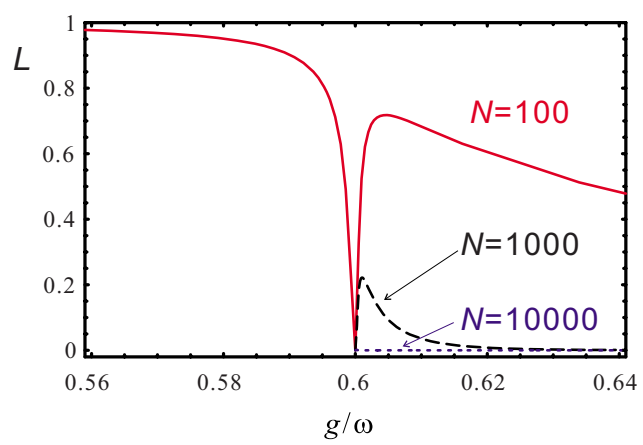

FIG. 4. (Color online) The LE of the systems for different $N$ at $\omega t=100$. In normal phase, the LE is independent of $N$. In superradiant phase, $N=100$ (solid line), 1000 (dashed line), and 10000 (dotted line), respectively, from up to bottom. For other parameters see Fig. 2. 
dust) flies into the cloud chamber, which is filled with supersaturated and supercooled water or alcohol, the water or alcohol vapor will condensate around the flying charged particle (or a dust) and form a liquid droplet, then a track is left. During this process, as a result of the sensitivity in response to the extra particle, the supersaturated vapor staying in the vicinity of the classical phase transition experiences a classical phase transition, transiting from vapor to liquid.

In the present investigation, similarly, there exists very sensitive dynamics of the photon-dressed atomic ensemble when a far-off-resonant atom goes through the cavity. In view of the Stark effect, the far-off-resonant atom shifts the frequency of the cavity field. We assume that the photondressed atomic ensemble is initially prepared in a state near the quantum critical point of the QPT of the Dicke model. Then the frequency change induced by the far-off-resonant atom will lead the Dicke model to cross the quantum critical point resulting in a sensitive dynamics of the LE. This quantum effect is similar to the classical phenomenon in the realistic cloud chamber that the vapor in the cloud chamber will condensate around the microscopic detected particle after experiencing the classical phase transition. Therefore, it is possible to realize the quantum version of the cloud chamber effect through observing the sensitive change in the LE of the photon-dressed atomic ensemble.

Here, the enhancement of the decay of LE or its sudden change can be regarded as an indicator of the one-atom induced QPT to detect the passage of the atom. This fact properly resembles the cloud chamber effect. In this analogy, the photon-dressed atomic ensemble, which can be tuned to the vicinity of the QPT point, behaves as the supersaturated vapor in the classical cloud chamber, while the enhancement of the decay of LE just resembles the transition from vapor to liquid.

Indeed, the LE in our paper is obtained from the decoherence factor for time evolution of the extra atom, but it actually represents the "mark" of this atom on the "cloud chamber"- the photon-dressed atomic ensemble. An obvious reason is that the LE only depends on the parameters of the "chamber" and, thus, is an intrinsic quantity of the chamber. Especially, the extra atom can only provide a small perturbation; thus, the LE is independent of the detected particle. In most of the references we cite, the LE can be defined without the detected particle by the chamber. It is only in our own paper [2] where the detected particle is introduced and it is proved that the decoherence factor of the detected particle is just the LE of the chamber. Thus, the LE is obviously the mark of the detected particle left in the chamber.

\section{CONCLUSION WITH A REMARK}

In summary, based on the QPT of the Dicke model, we have proposed a quantum critical model to display the ultrasensitivity of dynamic evolution of a QPT system of a photon-dressed atomic ensemble. We have also pointed out the analog of this one-atom induced QPT to the cloud chamber based on QPT. Frankly we have to point out that such a model cannot be implemented easily with the generic AMO system, since the two-photon term could not be simply ig- nored in the overstrong coupling limit [12]. However, our present study is still heuristic and the toy model covers the principle ideas for QPT inducing the cloud chamberlike effect. Furthermore, with the great development of solid quantum device physics, the Dicke model may be realized in some solid-state systems such as the superconducting quantum circuits and the nanomechanical resonators integrated with some qubit array systems.

Finally, we would like to mention a reference [18], in which an effective Dicke model was derived in a multilevel atomic ensemble. In this reference, the two-photon term $A^{2}$ may be safely ignored originally; thus, the modified Dicke model based on such a practical setup may be used to display the QPT phenomena we found in this paper.

\section{ACKNOWLEDGMENTS}

We would like thank Shuo Yang for helpful discussions. This work was supported by the National Natural Science Foundation of China with Grants No. 10935010 and No. 10775048, and the National Fundamental Research Program of China with Grants No. 2006CB921205 and No. 2007 CB925204.

\section{APPENDIX: COEFFICIENTS OF BOGOLIUBOV TRANSFORMATION}

\section{Normal phase}

The coefficients of Bogoliubov transformation in the normal phase are

$$
\begin{aligned}
& f_{1,2}=\frac{1}{2} \frac{\cos \theta}{\sqrt{\omega \omega_{A}}}\left(\omega \pm \omega_{A}\right), \\
& f_{3,4}=\frac{1}{2} \frac{\sin \theta}{\sqrt{\omega \omega_{B}}}\left(\omega \pm \omega_{B}\right),
\end{aligned}
$$

where the rotating angle in the coordinate-momentum representation $\theta$ is given by

$$
\tan 2 \theta=\frac{4 g \sqrt{\omega \omega_{0}}}{\omega_{0}^{2}-\omega^{2}} .
$$

\section{Super-radiant phase}

The coefficients of Bogoliubov transformation in the super-radiant phase are

$$
\begin{aligned}
& f_{1,2}^{\prime}=\frac{1}{2} \frac{\cos \theta^{\prime}}{\sqrt{\omega \omega_{A}^{\prime}}}\left(\omega \pm \omega_{A}^{\prime}\right), \\
& f_{3,4}^{\prime}=\frac{1}{2} \frac{\sin \theta^{\prime}}{\sqrt{\omega \omega_{B}^{\prime}}}\left(\omega \pm \omega_{B}^{\prime}\right),
\end{aligned}
$$

where the analogous rotating angle $\theta^{\prime}$ is

$$
\tan 2 \theta^{\prime}=\frac{2 \omega \omega_{0} \mu^{2}}{\omega_{0}^{2}-\mu^{2} \omega^{2}} .
$$


[1] S. Sachdev, Quantum Phase Transition (Cambridge University Press, Cambridge, 1999).

[2] H. T. Quan, Z. Song, X. F. Liu, P. Zanardi, and C. P. Sun, Phys. Rev. Lett. 96, 140604 (2006).

[3] J. Zhang, X. Peng, N. Rajendran, and D. Suter, Phys. Rev. Lett. 100, 100501 (2008).

[4] K. Hepp and E. H. Lieb, Ann. Phys. (N.Y.) 76, 360 (1973); Phys. Rev. A 8, 2517 (1973); Y. K. Wang and F. T. Hioe, ibid. 7, 831 (1973).

[5] C. Emary and T. Brandes, Phys. Rev. Lett. 90, 044101 (2003); Phys. Rev. E 67, 066203 (2003).

[6] P. Zanardi and N. Paunković, Phys. Rev. E 74, 031123 (2006).

[7] D. Rossini, T. Calarco, V. Giovannetti, S. Montangero, and R. Fazio, Phys. Rev. A 75, 032333 (2007).

[8] J. Zhang, F. M. Cucchietti, C. M. Chandrashekar, M. Laforest, C. A. Ryan, M. Ditty, A. Hubbard, J. K. Gamble, and R. Laflamme, Phys. Rev. A 79, 012305 (2009).

[9] L. C. Wang, X. L. Huang, and X. X. Yi, Phys. Lett. A 368, 362 (2007).

[10] R. H. Dicke, Phys. Rev. 93, 99 (1954).

[11] Z. P. Karkuszewski, C. Jarzynski, and W. H. Zurek, Phys. Rev.
Lett. 89, 170405 (2002); F. M. Cucchietti, D. A. R. Dalvit, J. P. Paz, and W. H. Zurek, ibid. 91, 210403 (2003); R. A. Jalabert and H. M. Pastawski, ibid. 86, 2490 (2001); T. Gorin, T. Prosen, T. H. Seligman, and M. Žnidarič, Phys. Rep. 435, 33 (2006)

[12] K. Rzazewski, K. Wódkiewicz, and W. Zacowicz, Phys. Rev. Lett. 35, 432 (1975).

[13] G. Liberti and R. L. Zaffino, Phys. Rev. A 70, 033808 (2004); Eur. Phys. J. B 44, 535 (2005); Y. Li, Z. D. Wang, and C. P. Sun, Phys. Rev. A 74, 023815 (2006); D. Tolkunov and D. Solenov, Phys. Rev. B 75, 024402 (2007); G. Chen, X. Wang, J. Q. Liang, and Z. D. Wang, Phys. Rev. A 78, 023634 (2008); Y. Li and Z. D. Wang, e-print arXiv:0904.4730.

[14] H. Fröhlich, Phys. Rev. 79, 845 (1950); Proc. R. Soc. London, Ser. A 215, 291 (1952); Adv. Phys. 3, 325 (1954).

[15] S. Nakajima, Adv. Phys. 4, 363 (1955).

[16] T. Holstein and H. Primakoff, Phys. Rev. 58, 1098 (1940).

[17] M. Wagner, Unitary Transformations in Solid State Physics (North-Holland, Amsterdam, 1986).

[18] F. Dimer, B. Estienne, A. S. Parkins, and H. J. Carmichael, Phys. Rev. A 75, 013804 (2007). 